
allemande

45-2 | 2013

Images et discours de la nation

\title{
Guillaume DUVAL, Made in Germany. Le modèle allemand au-delà des mythes
}

Paris, Seuil, 2013

Michel Hau

\section{(2) OpenEdition \\ Journals}

Édition électronique

URL : https://journals.openedition.org/allemagne/1823

DOI : 10.4000/allemagne. 1823

ISSN : 2605-7913

Éditeur

Société d'études allemandes

Édition imprimée

Date de publication : 30 décembre 2013

Pagination : 497-501

ISSN : 0035-0974

Référence électronique

Michel Hau, «Guillaume duval, Made in Germany. Le modèle allemand au-delà des mythes », Revue d'Allemagne et des pays de langue allemande [En ligne], 45-2 | 2013, mis en ligne le 29 juillet 2019, consulté le 18 mai 2021. URL : http://journals.openedition.org/allemagne/1823 ; DOI : https://doi.org/ 10.4000/allemagne.1823 
utilisés, pourtant nombreux). Par ailleurs, un certain nombre de constats fort justement opérés ne sont pas toujours mis en perspective avec la situation plus générale des rescapés de la Shoah, en Europe occidentale d'une part, dans l'Europe centrale passée sous la férule soviétique d'autre part. C'est ainsi que les remarques concernant la démographie et la sociologie des sociétés juives berlinoises, marquées par la sécularisation, les unions mixtes ou encore l'apparition d'un conflit générationnel à l'orée des années soixante ne sont que le reflet de tendances observables dans l'ensemble des communautés juives d'Europe au sens large, mais vaudrait aussi pour les États-Unis et sans doute même au delà des communautés confessionnelles, c'est l'ensemble des sociétés européennes qui est marqué après 1945 par ces phénomènes.

De la même manière, plusieurs aspects relatifs au fonctionnement des institutions communautaires, leur décalage avec la société juive qui critique ces "Juifs de métier » (p. 244) et l'atmosphère qui y règne, certes familiale mais également très paternaliste et patriarcale (p. 250), pourraient tout à fait s'appliquer à la situation observable dans les communautés juives en France ou en Pologne à la même époque, pour prendre deux exemples respectivement à l'Ouest et à l'Est de l'Europe. Une connaissance plus approfondie de la littérature anglo-saxonne - peu citée dans les notes et quasi absente de la bibliographie placée en fin de volume - aurait peut-être pu permettre d'élargir la perspective en replaçant notamment les spécificités est-allemandes dans un ensemble plus vaste incluant d'autres démocraties populaires où certaines problématiques entrent clairement en résonance (2). C'est ainsi que l'intéressante "renaissance » de l'attrait pour la culture et le patrimoine juifs, que l'auteur constate dans les années 1980 tant à l'Ouest qu'à l'Est s'observe également selon une chronologie parallèle et des modalités proches - notamment la restauration des cimetières juifs qui débute également en Pologne à cette période ${ }^{(3)}$.

Au total, et en dépit des quelques réserves émises, cette synthèse sur l'histoire des Juifs dans les deux Berlin depuis 1945 est incontestablement bienvenue dans l'historiographie en français encore trop pauvre sur l'histoire juive européenne après 1945. Elle apporte une somme de connaissances fort utiles tant dans une perspective comparatiste Est-Ouest que dans l'étude des communautés juives d'Europe rescapées de la Shoah.

Audrey KICHELEWSKI

Guillaume Duval, Made in Germany. Le modèle allemand au-delà des mythes, Paris, Seuil, 2013,234 p., $17 €$.

Cet ouvrage de quelqu'un qui a travaillé comme ingénieur pendant plusieurs années dans des entreprises allemandes mérite toute notre attention. Court et bien documenté sur de nombreux points, il aidera à combattre l'ignorance des Français au sujet de l'Allemagne, au moment où cette dernière fait sentir de plus en plus fortement son poids sur l'évolution de la société française. Toutefois, il n'échappe pas complètement à ces vues déformantes qui empêchent une grande partie de la classe politique française de comprendre les fondements actuels des rapports franco-allemands.

2 Parmi une littérature abondante, on peut citer Lynn RAPAPORT, Jews in Germany after the Holocaust: Memory, Identity and Jewish-German Relations, New York, Cambridge University Press, 1997, et Michael MENG, Shattered Spaces. Encountering Jewish Ruins in Postwar Germany and Poland, Cambridge, Harvard University Press, 2011, qui tous deux dépassent le cadre allemand pour inclure le cadre est-européen dans leur réflexion.

3 Sur cette question, cf. M. MENG, Shattered Spaces, op. cit., chapitre 4, "Restoring Jewish Ruins ». 
L'auteur montre l'ancienneté des facteurs qui fondent la réussite économique allemande. Sa thèse essentielle est qu'elle repose sur des causes structurelles et que "les profondes différences héritées de l'histoire continuent de marquer de façon déterminante nos sociétés et nos économies ». Sur ce point, l'ouvrage de Guillaume Duval est d'une grande richesse et il a de surcroît le mérite d'être clair.

Guillaume Duval a vu de l'intérieur tout ce que doivent les succès de l'industrie allemande à un mode de relations sociales reposant sur la cogestion. Les comités d'entreprise (Betriebsräte) donnent leur accord préalable à toute décision sur les horaires de travail, les congés, les recrutements et les promotions. Dès qu'une décision économique entraîne des conséquences sociales, l'employeur est tenu de présenter un plan social qui doit recueillir l'approbation du Betriebsrat, alors qu'en France, l'avis du comité d'entreprise $n$ 'est que consultatif. Les représentants des salariés occupent, depuis 1976, la moitié des sièges au conseil de surveillance des entreprises de plus de 2000 salariés. En contrepartie de cette participation à la gestion des entreprises, les syndicats de branche ont accepté une certaine flexibilité. En témoigne notamment l'introduction de clauses d'ouverture (Öffnungsklausel) dans les conventions collectives, ces clauses permettant aux entreprises en difficulté de déroger temporairement aux accords de branche. Comme l'Italie, I'Allemagne ne dispose pas de salaire minimum légal (principe dit de la «Tarifautonomie»). Le salaire minimum est fixé par la convention collective de la branche. On aura compris qu'il est plus bas dans le textile que dans la chimie. Les accords sont conclus pour une durée de deux ou trois ans, pendant laquelle les grèves sont interdites. Ces négociations de branche ont joué un rôle clé dans la stratégie menée pour défendre la compétitivitécoût de l'industrie allemande depuis la guerre. En France, remarque Guillaume Duval, il faut un niveau plus élevé de chômage et de liquidations d'entreprises pour modérer les revendications salariales.

Un autre avantage comparatif ancien de l'économie allemande est le respect que la société tout entière voue à la compétence professionnelle, quel que soit le domaine où elle s'exerce. En Allemagne, les emplois industriels sont nettement plus valorisés socialement qu'en France. L'apprentissage, au lieu d'être une filière de second choix, est la voie prédominante et n'est pas synonyme de formation courte. Même si, comme dans tous les pays riches aujourd'hui, les jeunes Allemands ont tendance à se détourner des études scientifiques et techniques, leur orientation vers les métiers de l'industrie reste plus forte qu'en France. De réelles possibilités de promotion interne sont ouvertes à ceux qui ont démarré leur vie professionnelle dans le cadre de l'apprentissage. Jürgen Schrempp, président de Daimler de 1995 à 2005, commença comme apprenti mécanicien, rappelle Guillaume Duval. Même si l'apprentissage rencontre aujourd'hui des difficultés, comme la réduction du nombre de places offertes par les entreprises, il permet à l'Allemagne ainsi qu'aux pays ayant des systèmes d'enseignement similaires (Autriche, Pays-Bas, auxquels on peut ajouter la Suisse) d'avoir en 2011 un taux de chômage des 15-24 ans inférieur à $10 \%$ des actifs contre plus de $20 \%$ en France. Ce rapport du simple au double se maintient même si l'on considère l'ensemble de la classe d'âge: en 2011, les jeunes chômeurs représentaient 8,4 \% des jeunes en France contre 4,5\% en Allemagne.

La longue survie des corporations, jusque pendant le xixe siècle, a permis de développer la coopération interentreprises au sein d'une même branche d'activité, notamment dans le domaine de la formation professionnelle et de la définition de normes industrielles. Très contraignantes, ces dernières constituent aujourd'hui un avantage comparatif pour l'industrie allemande: les règles du Deutsches Institut für Normung ont été la source d'économies d'échelle considérables en permettant une standardisation plus poussée et elles ont allégé les coûts de transaction en limitant les incertitudes sur la qualité des produits. Elles ont été largement reprises par les normes européennes... auxquelles les entreprises allemandes sont - et pour cause - celles qui s'adaptent le plus facilement. La France, pays plus libéral que l'Allemagne sur ce plan, a souffert de l'individualisme de ses entreprises et de l'absence d'un rôle substantiel confié aux branches professionnelles. 
D'autres avantages structurels sont encore à prendre en compte. La densité du peuplement se traduit par un coût moindre des réseaux. La fragmentation politique de l'Allemagne a, comme en Italie du Nord, favorisé l'esprit d'entreprise. Sur ce dernier point, Guillaume Duval note que ce qui fut longtemps perçu comme un handicap se révèle au bout du compte comme un avantage décisif dans un contexte de mondialisation où les petits États et les États décentralisés tirent mieux leur épingle du jeu.

Guillaume Duval montre que les Allemands sont préparés depuis longtemps au nouvel ordre international fondé sur le libre jeu du marché et le rejet du dirigisme. L'ordolibéralisme, qui s'est imposé en 1947, avec le choix du professeur Ludwig Erhard pour assumer la responsabilité des questions économiques de la bizone, est en réalité un courant de pensée développé dès le lendemain de la Première Guerre mondiale par les économistes de l'université de Fribourg-en-Brisgau. Il considère les mécanismes de marché comme plus efficaces que l'économie administrée, tout en posant, à la différence du libéralisme anglosaxon, que l'État doit établir un certain nombre de règles. Les idées de l'ordolibéralisme ont été provisoirement écartées avec l'arrivée de Hitler au pouvoir et la remise en place d'une économie de guerre, administrée par l'État. C'est pourquoi les Allemands associent l'idée de relance keynésienne et d'intervention de l'État à la politique de réarmement à marche forcée et à la catastrophe hitlérienne. Le succès des thèses ordolibérales en Allemagne depuis 1945 s'explique par la défiance qu'éprouve l'opinion publique à l'égard de toute forme d'intervention étatique, à la suite du traumatisme laissé par l'expérience nazie. Guillaume Duval note que ce rejet de l'interventionnisme étatique a été renforcé par la répression sanglante des émeutes populaires à Berlin-Est en 1953, alors qu'il a fallu attendre la fin des années 1970 pour que le modèle soviétique soit définitivement discrédité en France. Le rejet du dirigisme par l'Allemagne ne signifie pas la renonciation à conduire des projets industriels à long terme: l'absence de ce que les Français appellent une "politique industrielle » est compensée par la coopération interentreprises, fondée sur une connaissance fine du terrain.

Plus récemment, de nouveaux avantages comparatifs sont venus s'ajouter aux avantages structurels anciens: l'absence de bulle immobilière liée à la stagnation du nombre d'habitants, une dépense publique plus faible due à une moindre prise en charge des jeunes enfants ainsi qu'au caractère sélectif de l'accès à l'université et enfin la compression des prix des services, où les salaires horaires sont en moyenne un tiers plus bas qu'en France; trois millions de salariés allemands, pour l'essentiel, des femmes travaillant dans le secteur tertiaire, gagnent moins de 6 euros de l'heure en 2010 (alors que le SMIC horaire est de 8,90 euros pour toutes les branches en France). Ce bas prix des services a joué un rôle dans l'amélioration de la compétitivité-coût de l'industrie allemande, en rendant plus supportable la stagnation des rémunérations des salariés de l'industrie, mais la crise a accru l'inégalité de temps de travail entre les hommes et les femmes aux dépens de ces dernières. À ces nouveaux avantages comparatifs se sont ajoutés, depuis 1990, la chute du communisme, qui rend possible une symbiose industrielle profitable avec les ex-pays frères de la défunte RDA, ainsi que le décollage des pays émergents, dont la demande se trouve en accord avec les spécialisations traditionnelles de l'industrie allemande.

Là où il est moins convaincant, c'est dans sa critique radicale des mesures prises par Gerhard Schröder, qui constitue la seconde thèse centrale de son ouvrage. Non seulement celles-ci n'auraient eu aucune part dans les succès actuels de l'économie allemande, mais encore elles l'auraient plutôt fragilisée à long terme. Dès l'introduction, l'auteur laisse paraître son hostilité au néo-libéralisme. Il condamne d'un trait de plume le système économique des pays anglo-saxons "démonétisé, dit-il, par les frasques de ses financiers". Mais si l'on veut bien se donner la peine de comparer le taux moyen de croissance du PIB français depuis quatre décennies à ceux des États-Unis et de l'Angleterre, la comparaison 
est à l'avantage de ces derniers. Entre 1974 et 2008, le PIB de la France a été multiplié par 2, celui du Royaume-Uni par 2,3 et celui des États-Unis par 2,8. Les taux de croissance annuels moyens sont respectivement de 2,1\%, 2,4\% et 3,1\% par an ${ }^{(4)}$.

Le chapitre consacré à la gestion de Gerhard Schröder est sous-titré: "anatomie d'une mystification ». II reproche au chancelier social-démocrate d'avoir développé la pauvreté et les inégalités. Et de comparer les performances allemandes et françaises en matière de redistribution de revenus: le taux de pauvreté ${ }^{(5)}$ allemand est passé de 10,5\% en 1999 à 14,5 \% en 2006 et le rapport entre les revenus des 10 \% les plus riches et ceux des $10 \%$ les plus pauvres est passé de 5,3 en 1997 à 7,1 en 2006, quand en France il retombait de 7,7 à 6,1 grâce à la forte hausse du Smic horaire due aux 35 heures. En fait, il y a là une profonde divergence entre les intellectuels des deux pays sur la notion de pauvreté. Pour les Français, celle-ci se mesure uniquement en termes monétaires. C'en est au point que les experts français des reconversions industrielles ont considéré le problème résolu quand ils avaient multiplié les préretraites et les congés de fin de carrière. Or les populations sorties des chiffres du chômage et entrées dans la catégorie des non actifs ont sombré dans les addictions, la perte des repères sociaux, et les troubles de santé. Ce fait n'a jamais fait l'objet d'analyses statistiques poussées: il est bien dommage qu'il n'existe pas de médecine de l'oisiveté à côté de la médecine du travail. En faisant passer la durée d'indemnisation du chômage de 32 à 12 mois, en rendant plus stricte l'obligation d'accepter les emplois proposés, en imposant les « ein Euro jobs » aux chômeurs de longue durée et en exonérant de charges sociales les "minijobs » à temps partiel de moins de 400 euros par mois, le gouvernement social-démocrate a redonné un emploi du temps à des centaines de milliers de personnes fragilisées psychologiquement par l'inactivité. Pour un Allemand, la condition de "working poor » est moins dérangeante que celle de chômeur et d'assisté. Cette divergence fondamentale fait que l'expérience Schröder de baisse de la rémunération du travail (qualifiée par Guillaume Duval de «multiplication des petits boulots mal payés ») est rejetée avec indignation par une bonne partie de l'opinion française alors qu'elle est vue de façon moins négative par l'opinion allemande.

Guillaume Duval met l'accent sur l'effet récessif immédiat des mesures prises par le gouvernement Schröder. II cite en abondance les statistiques qui enregistrent la décélération de la croissance, la contraction de la demande intérieure et la baisse des investissements des entreprises pendant la période 1998-2005. Mais la baisse des coûts unitaires de main-d'œuvre enregistrée en Allemagne entre 1998 et 2006 n'est évoquée que très incidemment, en quelques lignes. Or c'est le redémarrage des exportations qui a permis à l'économie allemande de renouer durablement avec la croissance. Au même moment, la croissance française, qui avait trop longtemps reposé sur la dépense publique, a fini par buter sur le bon vouloir des créanciers étrangers. L'auteur a bien connaissance du «redressement indéniable » de l'économie allemande après 2005, mais il affirme qu'il " doit en réalité fort peu à l'action de Gerhard Schröder ». On aimerait alors savoir quels autres facteurs expliquent cette réussite, mais le chapitre suivant ("Les vraies raisons du rebond de l'Allemagne d'Angela Merkel ») laissera le lecteur sur sa faim: l'auteur se contente en fait de rappeler les avantages comparatifs structurels et conjoncturels de l'économie allemande. Sa foi dans le keynésianisme est si fervente qu'elle lui fait même entrevoir une vision dans laquelle l'austérité imposée par Schröder se serait "quelque peu relâchée avec Angela Merkel ». Il en oublie, dans ce chapitre, la décision de la chancelière d'augmenter de trois points la TVA, passée d'un seul coup de

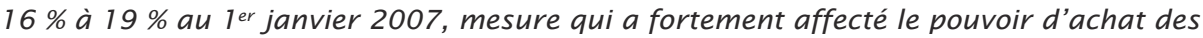
Allemands. S'il est vrai que la part des salaires est remontée légèrement dans la valeur

4 Cf Angus MADDISON, Statistics on World Population, GDP and per capita GDP, 2-2010 AD, http://www.ggdc.nl/maddison.

5 Proportion de la population vivant au-dessous d'un niveau de revenu égal à $60 \%$ du revenu médian. 
ajoutée entre 2007 et 2012, c'est à l'amélioration de la santé des entreprises et à la baisse du chômage qu'on le doit, et non à un assouplissement de la politique économique du gouvernement fédéral.

Au total, ce livre, même s'il aide à dissiper beaucoup de préjugés erronés sur l'Allemagne, ne contribue pas encore à éclairer suffisamment les Français sur la situation exacte de leur pays dans le couple franco-allemand. Il comporte un certain nombre d'omissions et de non-dits. Il souffre sans doute d'être biaisé par une pieuse défense de la politique française traditionnelle de soutien de la croissance par la demande intérieure. Pourtant, la preuve par l'absurde est depuis longtemps sous nos yeux: une lente asphyxie du potentiel productif français qui se manifeste par la coexistence d'un déficit commercial extérieur croissant et d'un sous-emploi massif.

Michel HaU 